\title{
Behavior of zonal components of CR distribution during periods of solar wind disturbances
}

\section{P. Yu Gololobov*, V.G. Grigoryev, S.A. Starodubtsev}

The Yu.G. Shafer Institute of Cosmophysical Researches and Aeronomy, Yakutsk, Russia

E-mail: gpeter@ikfia.ysn.ru, grig@ikfia.ysn.ru, starodub@ikfia.ysn.ru

\begin{abstract}
On the basis of processing of world-wide neutron monitor network data (NMDB http://www.nmdb.eu) using the global survey method in real time, a behaviour of the first two angular moments of a cosmic ray distribution function for the period 2015-2016 are investigated. As a result of the investigation new criteria of behaviour of their parameters before beginnings of geomagnetic disturbances are found. Also the relationship between the manifested predictors and variations of Dst- and Kp-indexes are considered. It is shown that in some cases there is a connection between abrupt increases of positive values of the zonal components and increases of Kp-index values, which is due to the auroral phenomena in the sub-polar regions of the Earth. The results of the investigation can be used for more effective space weather forecasting.
\end{abstract}




\section{Introduction}

The method of global survey developed at the ShICRA SB RAS [1], which is based on measurements of the world-wide network of neutron monitors, allows to precisely define the first 9 components of function of angular distribution of cosmic rays (CR) for each hour of observations. Creation and development of the neutron monitor network database NMDB (http://www.nmdb.eu) enables using the method in real-time mode [2] that is of considerably interest for space weather forecasting. In the work [2], the behavior of radial $X_{11}$ and azimuthal $Y_{11}$ components of the first harmonic of angular distribution (or diurnal anisotropy) of galactic CR before the beginning of geomagnetic storm with Dst $\leq-50 \mathrm{nT}$ was investigated. It was found that in the time interval from couple hours to 2 days before the beginning of most geomagnetic storms the components of diurnal anisotropy vector revealed distinctive changes which were not observed during periods of quiet solar wind. Hence using in practice of these geomagnetic disturbance predictors has a serious lack, the same changes also appears when a solar wind disturbance which doesn't cause the storm comes close to the Earth. Later in the works $[3,4]$ it was shown that more effective predictors of geomagnetic storm beginnings are dynamics of changes of amplitudes of zonal components of CR distribution function.

In the current work, on the basis of global survey method, the relationship between behavior of zonal components of isotropic part $C_{00}$, diurnal $C_{10}$ and semi-diurnal $C_{20}$ variations of CR intensity and geomagnetic storm appearances, which were observed in 2015-2016, is investigated.

\section{Experimental data}

In result of analysis of the behavior of mentioned components we found that the excess of the positive values of amplitudes of components $C_{00}, C_{10}$ and $C_{20}$ over the values $0.5 \%, 0.9 \%$ and $0.9 \%$, respectively, can be considered as the effective predictor of geomagnetic storm beginnings. Also the predictor of geomagnetic storm was the excess of the sum of these components over the value $1.1 \%$. In table the dates of appearance of the mentioned predictors, which are determined by the global survey method, and observations of geomagnetic storms with Dst $<-38 \mathrm{nT}$ for the studied time period (WDC for Geomagnetism, Kyoto, http://wdc.kugi.kyoto-u.ac.jp/index.html) are presented. Additionally, the dates of beginnings of Kp-index increase over 4 which didn't relate to a geomagnetic storm, but had any of the mentioned predictors, are listed. The analysis of the table leads us to the next conclusions:

- 9 magnetic storms with Dst $\leq-100 \mathrm{nT}$ had at least one of the predictors with a lead time form 1 to 22 hours;

- from 26 magnetic disturbances with the amplitudes $-100 \mathrm{nT} \leq$ Dst $\leq-38 \mathrm{nT}$, 6 events didn't have any predictor;

- manifestations of 10 predictors weren't accompanied with geomagnetic storms, but their appearance preceded or coincided with the value of $\mathrm{Kp}>4$.

- 9 observed «false» predictors weren't accompanied by geomagnetic storms and increase of Kp-index, but were caused by small perturbations of solar wind. 
Thus, the results of analysis of amplitude changes of the zonal harmonics shows that around $80 \%$ of the considered events of geomagnetic storms had predictors in components $C_{00}$ or $C_{20}$ and rarely $C_{10}$ (2 events). 


\begin{tabular}{|c|c|c|c|c|c|c|c|}
\hline 39. & $03.06 .19^{\mathrm{h}}$ & - & $03.06 .08^{\mathrm{h}}$ & $03.06 .09^{\mathrm{h}}$ & $03.06 .18^{\mathrm{h}},-98 \mathrm{nT}$ & $10^{\mathrm{h}}$ & \\
\hline 40. & $03.11 .07^{\mathrm{h}}$ & - & - & - & - & $03.11 .12^{\mathrm{h}}$ \\
\hline 41. & - & - & - & - & $04.02 .18^{\mathrm{h}},-56 \mathrm{nT}$ & & \\
\hline 42. & - & - & $04.0716^{\mathrm{h}}$ & $04.07 .14^{\mathrm{h}}$ & $04.07 .20^{\mathrm{h}},-60 \mathrm{nT}$ & $06^{\mathrm{h}}$ & \\
\hline 43. & - & - & - & $04.19 .19^{\mathrm{h}}$ & - & & \\
\hline 44. & $04.20 .09^{\mathrm{h}}$ & - & - & - & - & & \\
\hline 45. & $05.06 .16^{\mathrm{h}}$ & - & - & $05.06 .18^{\mathrm{h}}$ & $05.08 .03^{\mathrm{h}},-83 \mathrm{nT}$ & $35^{\mathrm{h}}$ & \\
\hline 46. & $05.16 .15^{\mathrm{h}}$ & - & - & - & - & - & \\
\hline 47. & - & - & $06.05 .06^{\mathrm{h}}$ & - & $06.05 .20^{\mathrm{h}},-46 \mathrm{nT}$ & $14^{\mathrm{h}}$ & \\
\hline 48. & $06.22 .17^{\mathrm{h}}$ & - & - & - & - & - & $06.22 .18^{\mathrm{h}}$ \\
\hline 49. & $07.19 .17^{\mathrm{h}}$ & - & - & $07.19 .23^{\mathrm{h}}$ & $07.20 .00^{\mathrm{h}},+49 \mathrm{nT}$ & $07^{\mathrm{h}}$ & \\
\hline 50. & - & $08.03 .01^{\mathrm{h}}$ & - & - & $08.03 .06^{\mathrm{h}},-51 \mathrm{nT}$ & $05^{\mathrm{h}}$ & \\
\hline 51. & $08.23 .15^{\mathrm{h}}$ & - & - & - & $08.23 .14^{\mathrm{h}},-74 \mathrm{nT}$ & & \\
\hline 52. & $09.15 .20^{\mathrm{h}}$ & - & - & - & - & & \\
\hline 53. & $09.17 .03^{\mathrm{h}}$ & - & - & - & - & & \\
\hline 54. & - & - & - & $10.13 .04^{\mathrm{h}}$ & $10.13 .08^{\mathrm{h}},-104 \mathrm{nT}$ & $04^{\mathrm{h}}$ & \\
\hline 55. & $11.10 .15^{\mathrm{h}}$ & - & - & - & $11.10 .12^{\mathrm{h}},-59 \mathrm{nT}$ & & \\
\hline 56. & - & - & $12.05 .07^{\mathrm{h}}$ & $12.05 .07^{\mathrm{h}}$ & & - & \\
\hline
\end{tabular}

* - means the absence of precursors of geomagnetic storm with Dst $\leq-38 \mathrm{nT}$.

As already was mentioned above, there were observed events when appearance of the predictors weren't accompanied with geomagnetic storms, but were associated with the increase of $\mathrm{Kp}>4$. Wherein it was found that this periods were mainly accompanied with the abrupt increase of zonal component $C_{00} \geq 0.5 \%$. When $\mathrm{Kp} \geq 4$ the increase of $C_{00}$ happens with a lead time from several hours up to a day or sometimes almost simultaneously. In figure 1 the behavior of the $C_{00}$ value, Kp- and Dst-indices which were observed in June (a) and July (b) 2017. The straight lines shows the critical values of $C_{00}$ and Kp-index that have been taken by us. It can be noted from the figure 1 that exceeding of these levels is observed in the complete absence of perturbations of Dst-index. Geo-effective disturbances of solar wind in the sub-polar regions of the Earth are accompanied with increase of Kp-index, that is sensitive to different
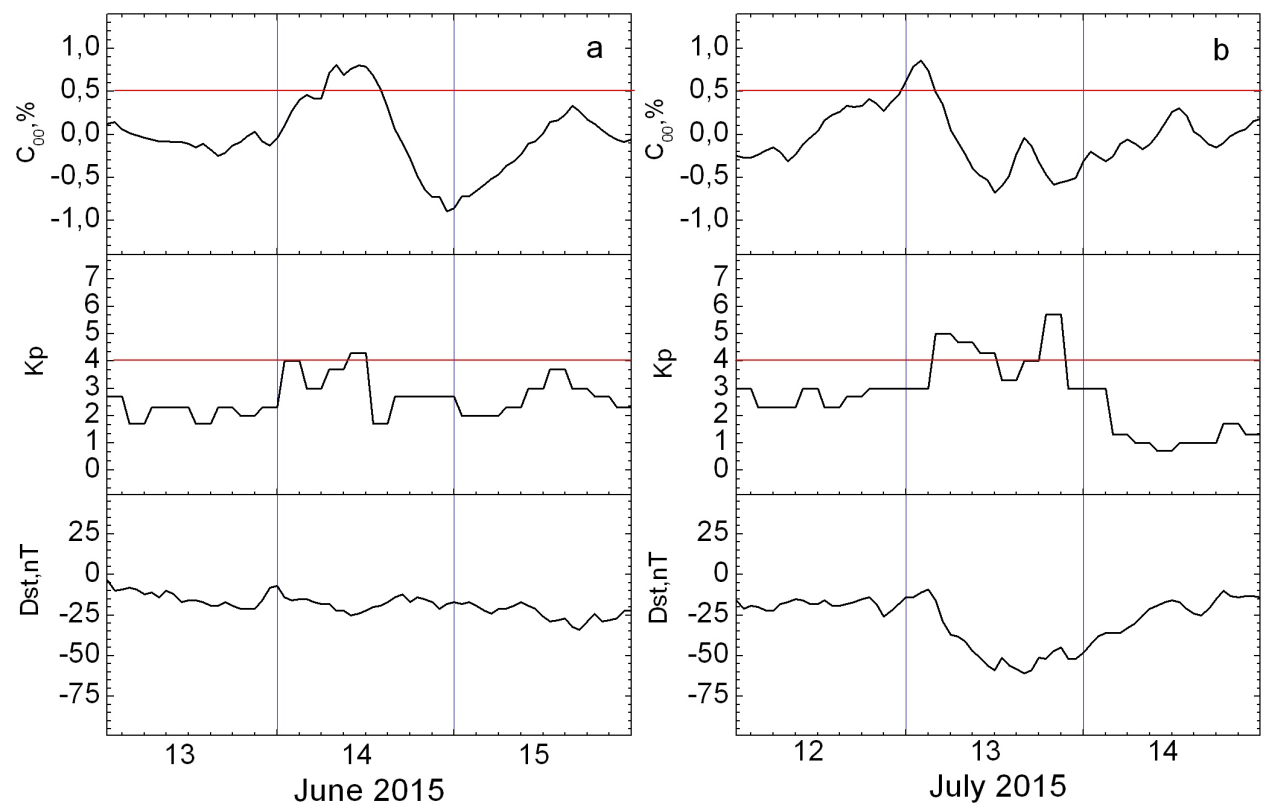

Figure 1: Time dependence of the values $C_{00}$, Kp-index and Dst-index in June (a) and July (b) 2015. 
magnetosphere-ionospheric current systems. Exceeding of the Kp-index values over 4 can be related in this case to auroral phenomena (magnetic sub-storms in the sub-polar regions of the Earth) and wherein variations of Dst-index can be insignificant. It is necessary to conduct further investigations that would show possibility of real use of the current parameter of zonal component $C_{00}$, which is defined in real-time, as a predictor of geo-effective disturbances in the sub-polar regions of the Earth.

\section{Conclusion}

On the basis of the conducted investigation one can make the next conclusions:

1. For the considered time period 2015-2016 the critical parameters of abrupt changes of the values of zonal harmonics of CR distribution which most really reflect the approach of geoeffective disturbances of solar wind are defined. At the current moment taking into account the obtained results we continue continuous ground-based monitoring of CR for the purpose of forecasting space weather, which results are available in the Internet through the web site http://www.ysn.ru/ starodub/SpaceWeather/global survey real time.html.

2. The relationship between increases of Kp-index over a value 4 and increases of the values of zonal components of the isotropic component $\left(C_{00} \geq 0.5 \%\right)$ and the second zonal harmonic $\left(C_{20}>0.9 \%\right)$ of $\mathrm{CR}$ angular distribution during quiet and weak geomagnetic field disturbance level (Dst $\leq-50 \mathrm{nT}$ ) is shown. The possible reason of this relationship is manifestations of magnetic sub-storms in the sub-polar regions of the Earth.

\section{Acknowledgments}

The work was supported by the grants RFBR Nos. 15-42-05085-r_vostok_a, 15-42-05083r_vostok_a and the program of Presidium of SB RAS No. 23. We acknowledge the NMDB database (http://www.nmdb.eu), founded under the European Union's FP7 programme (contract no. 213007) for providing data and we also acknowledge WDC for Geomagnetism (Kyoto) for providing the data of Dst-index of the geomagnetic field. The OMNI data were obtained from the GSFC/SPDF OMNIWeb interface at https://omniweb.gsfc.nasa.gov.

\section{References}

[1] A.M. Altukhov, G.F. Krymsky, A.I. Kuzmin, The method of "global survey” for investigating cosmic ray modulation, Proc. 11-th ICRC 4 (1970) 457.

[2] V.G. Grigoryev and S.A. Starodubtsev, Global Survey Method in Real Time and Space Weather Forecasting, Bull. of the RAS: Physics 79 (2015) 649.

[3] V.G. Grigoryev, S.A. Starodubtsev, P.Yu. Gololobov, Dynamics of zonal components of cosmic ray distribution during geomagnetic storm periods, in proceedings of 34-th ICRC PoS(ICRC2015)076.

[4] V.G. Grigoryev, S.A. Starodubtsev, P.Yu. Gololobov, Monitoring geomagnetic disturbance predictors using data of ground measurements of cosmic rays, Bull. of the RAS: Physics 81 (2017) 200. 